\title{
Enhancement of ATP generation capacity, antioxidant activity and immunomodulatory activities by Chinese Yang and Yin tonifying herbs
}

\author{
Kam Ming Ko* and Hoi Yan Leung
}

Address: Department of Biochemistry, Hong Kong University of Science and Technology, Clear Water Bay, Hong Kong, China

Email: Kam Ming Ko* - bcrko@ust.hk; Hoi Yan Leung - bclhy@ust.hk

* Corresponding author

Published: 27 March 2007

Chinese Medicine 2007, 2:3 doi:10.1186/1749-8546-2-3

This article is available from: http://www.cmjournal.org/content/2/l/3

(c) 2007 Ko and Leung; licensee BioMed Central Ltd.

This is an Open Access article distributed under the terms of the Creative Commons Attribution License (http://creativecommons.org/licenses/by/2.0), which permits unrestricted use, distribution, and reproduction in any medium, provided the original work is properly cited.

\begin{abstract}
Chinese tonifying herbs such as Herba Cistanche, Ganoderma and Cordyceps, which possess antioxidant and/or immunomodulatory activities, can be useful in the prevention and treatment of age-related diseases. Pharmacological studies on Yang and Yin tonifying herbs suggest that Yang tonifying herbs stimulate mitochondrial adenosine triphosphate (ATP) generation, presumably through the intermediacy of reactive oxidant species, leading to the enhancement of cellular/ mitochondrial antioxidant status. Yin tonifying herbs, however, apart from possessing antioxidant properties, exert mainly immunomodulatory functions that may boost a weak immune system and may also suppress overreactive immune responses. The abilities of Yang and Yin Chinese tonifying herbs to enhance ATP generation and to exhibit antioxidant and/or immunomodulatory actions are the pharmacological basis for their beneficial effects on the retardation of aging.
\end{abstract}

\section{Background}

Aging is a process of bodily change with time, leading to increased susceptibility to disease, and ultimately death. Because reactive oxidant species (ROS) and immune dysfunction are major causes of age-related diseases [1-3], the maintenance of antioxidant and immune fitness is a rational approach to preventive health care. Accumulation of ROS-induced oxidative damage to DNA, proteins, and other macromolecules has been regarded as a major endogenous cause of aging [1]. In addition to ROS-mediated cellular damage, aging was found to be associated with immune senescence, attributable at least partly to the loss of $\mathrm{T}$ lymphocyte functions [2,3]. Such loss increases the prevalence of infectious diseases in the elderly. With advances in modern medical research techniques, research on age-related chronic illnesses has become intense, in the quest for valuable preventive and therapeu- tic measures. Humans have been making continuous efforts to fight aging. As Chinese medicine has always emphasized the prolongation of a healthy lifespan, many Chinese tonifying herbs have long been used to safeguard health and to delay the onset of senility.

Under both normal and pathological conditions, ROS are generated in all cells undergoing aerobic metabolism, particularly from mitochondria. The cell possesses two distinct antioxidant defense systems to counteract damaging ROS: (1) enzymatic antioxidants such as catalase, superoxide dismutase (SOD), glutathione peroxidase and other related enzymes/molecules, and (2) non-enzymatic antioxidants such as ascorbic acid (vitamin C), $\alpha$-tocopherol (vitamin E) and $\beta$-carotene. To achieve optimal antioxidant fitness, every component of the antioxidant defense system should function optimally because antioxidants 
must work together in a synergistic manner. Chinese tonifying herbs have been shown to possess both in vitro and in vivo antioxidant activities $[4,5]$.

The immune system fights against 'foreign invaders' such as bacteria, viruses, fungi, yeasts and parasites. The humoral and cell-mediated immune responses show great competence in dealing with intruders. Moreover, the surveillance function of the immune system tends to prevent cancers, particularly in old age. However, an overreactive or imbalanced immune system can cause allergies or autoimmune disorders. A well-constituted and balanced immune system is thus crucial for safeguarding health. Chinese tonifying herbs have been shown to stimulate or suppress the cell-mediated immune response both in vitro and in vivo [6].

The importance of disease prevention has been recognized by Chinese medicine through experience accumulated over centuries. Many Chinese tonifying herbs have long been used for safeguarding health and for delaying the onset of senility. According to Chinese medicine theories, tonifying herbs prescribed for various symptoms of ill-health are generally classified into four categories on the basis of their health-promoting actions, namely 'Yanginvigorating', the 'Qi-invigorating', the 'Yin-nourishing' and the 'Blood-enriching' herbs [7]. The 'Qi-invigorating' and 'Blood-enriching' herbs are of Yang and Yin characteristics respectively. Chinese medicine theories suggest that a balance of Yin and Yang is essential to sustain optimal body function [8]. From a modern medical perspective, the maintenance of Yin and Yang in harmony may be described as the attainment of bodily homeostasis. The long-known antagonistic relationship between parasympathetic and sympathetic neural activities affords an example of both a phenomenon well-recognized by Western medicine and the Yin/Yang balance. A recent psychophysiological investigation in humans revealed an association between decreased parasympathetic or sympathetic activities with deficiencies of Yin or Yang respectively [9].

The theoretical framework of Chinese medicine is based on the Chinese cultural fabrics and clinical experience, while modern Western medicine has been established on the basis of laboratory and clinical investigations [10]. As the two distinct medical systems are complementary, bridging of the knowledge gap between Chinese and Western medicine is essential for their integration, in clinical practice, for disease prevention and treatment. Expounding Chinese medicinal theories in modern scientific terms to a Western audience facilitates communication between practitioners of the two systems.
In our earlier studies, we found that tonifying herbs with Yang or Yin properties were associated with antioxidant and immunostimulatory activities respectively [4]. Recent studies indicated that only Yang tonifying herbs (not Yin tonifying herbs) enhanced mitochondrial ATP generation capacity in mouse hearts [11]. We therefore suggest that Yang tonifying herbs enhance mitochondrial ATP generation, while Yin tonifying herbs are associated with immunomodulatory activities. In this mini-review, we summarize the abilities of Yang and Yin tonifying herbs to enhance ATP generation capacity, and to potentiate antioxidant and/or immunomodulatory actions, in an effort to characterize their respective pharmacological properties.

\section{Enhancement of ATP generation by Yang tonifying herbs}

In Chinese medicinal theories, Yang is a manifestation of body functions supported by various organs. A 'Yanginvigorating' action therefore involves the enhancement of bodily functions in general and cellular activities that consume ATP in particular. The mitochondrion is responsible for the generation of ATP through oxidative metabolism. To establish the pharmacological basis of 'Yanginvigorating' action, we have recently investigated the effect of Yang herbs on ATP generation capacity in heart homogenates prepared from mice that were pretreated with methanolic extracts of herbs [11]. Tonifying herbs from other functional categories were examined for comparison. While Chinese herbs are usually extracted by water for human oral consumption, water was replaced by methanol in our study for convenience in the processing and storage of samples. Yang herbs invariably enhanced myocardial ATP generation, with stimulation ranging from 20-130\%. Herba Cynomorii and Semen Cuscutae were the most potent herbs examined. By contrast, none of the Yin herbs enhanced ATP generation; some Yin herbs even suppressed ATP generation slightly (Table 1). A preliminary mechanistic study indicated that Yang herbs may speed up ATP synthesis by increasing mitochondrial electron transport [11].

\section{Correlation between enhancement of ATP generation capacity and antioxidative capacity}

Mitochondrial oxidative phosphorylation generates ROS as byproducts. Highly reactive chemically, ROS attack cellular structures located near the sites where ROS are generated. Mitochondrial DNA, proteins, and lipids in the inner membrane of mitochondria are thus vulnerable to oxidative damage [12], resulting in generalized organelle dysfunction, defective mitochondrial biosynthesis and poor energy metabolism [13].

Under normal physiological conditions, the mitochondrial antioxidant defense system adequately handles the potentially detrimental effects of ROS derived from energy 
Table I: Effect of Yang and Yin tonifying herbs on myocardial ATP generation capacity in mice ex vivo

\begin{tabular}{lccc}
\hline Herbs & ATP generation $(\%$ control) & Herbs & ATP generation (\% control) \\
\hline Yang herbs & & Yin herbs & $95.5 \pm 12.1$ \\
Cortex Eucommiae & $157 \pm 11.2 *$ & Semen Prinsepiae & $101 \pm 5.00$ \\
Herba Cistanches & $191 \pm 3.20 * *$ & Fructus Ligustri & $102 \pm 4.53$ \\
Herba Cynomorii & $230 \pm 17.3 * *$ & Rhizoma Polygonati & $98.2 \pm 9.92$ \\
Rhizoma Curculiginis & $149 \pm 38.0$ & Radix Asparagi & $102 \pm 8.51$ \\
Herba Epimedii & $130 \pm 7.51 *$ & Radix Ophiopogonii & $85.0 \pm 7.43$ \\
Radix Dipsaci & $120 \pm 19.7$ & Radix Oryzae & $82.7 \pm 0.92 *$ \\
Rhizoma Drynariae & $154 \pm 14.6 *$ & Herba Dendrobii & $80.3 \pm 7.05 *$ \\
Fructus Psoraleae & $175 \pm 13.4 *$ & Herba Ecliptae & \\
Semen Cuscutae & $222 \pm 2.12 * *$ & & \\
Radix Morindae & $142 \pm 19.3$ & & \\
Semen Allii & $133 \pm 35.8$ & & \\
\hline
\end{tabular}

Mice were pretreated with herbal extracts at daily doses of I g/kg for 3 days. The mean value of myocardial ATP generation capacity in unpretreated mice was $147 \pm 17.6$ (S.D.) nmol ATP/mg protein/10 min, $(n=6)$. $* P<0.05$; $* *<<0.01$, Student's $t$ test

metabolism [14]. When a functional imbalance between ROS levels and antioxidant concentrations caused by various disease states and/or aging occurs, age-related disorders such as cancer, cardiovascular diseases, brain dysfunction, or cataract may occur [15]. Antioxidant supplementation, particularly from herbal extracts, has become a trend in preventive health care.

Using an oxygen radical absorbance capacity assay, Ou et al. recently compared the free radical scavenging (i.e. antioxidant) activities of Yang and Yin herbs [16]. The results indicated that Yin herbs generally possessed higher antioxidant activities than Yang herbs and that the antioxidant potencies correlated well with the amounts of total phenolic compounds in the herbs. The authors suggested an analogy between Yin/Yang balance and antioxidation/ oxidation in energy metabolism. These findings of higher antioxidant activities in Yin herbs as compared with those in Yang herbs do not agree with the findings from one of our earlier studies which showed that most of the Yang herbs possessed a more potent 1,1-diphenylpicryhydrazyl radical-scavenging action than other tonifying herbs [4] (Table 2). Although the use of different herbal extraction methods and distinct antioxidant assays precludes direct comparison of the two studies, the discrepancy might be due to the selection of almost completely different sets of Yin and Yang herbs for testing in the two studies. Our study focused on herbs used for safeguarding health (i.e. herbs used for tonifying purposes) (Tables 2, Table 3 of reference [17]). Ou et al. probably used a selection criterion based on the general Yin and Yang properties of the herbs instead of their Yin-tonifying and Yang-tonifying actions [16]. Szeto and Benzie, using the same set of herbs described in $\mathrm{Ou}$ et al. to examine possible protective effects on DNA oxidative damage, found that the Yang herbs showed an antioxidant effect superior to that of Yin herbs [5].

\section{Antioxidant activities of Yang tonifying herbs}

Several Yang herbs have been shown to possess antioxidant activities both in vitro and in vivo (Table 4). In vitro free radical-scavenging activities were detected in herbal extracts prepared from Herba Epimedii [4,18], Radix Dipsaci [4,16], Fructus Psoraleae [4], Semen Cuscutae [16], Herba Cistanche [4,16,18], Cortex Eucommiae [19] and Rhizoma Cibotii $[4,16]$. Aqueous extracts of Rhizoma Drynariae and Cortex Eucommiae were found to inhibit oxidant production from rat osteoblasts [20], and also inhibited biomolecular oxidative damage [21]. Active ingredients (bakuchiol, isobavachin and isobavachalcone) from Fructus Psoraleae inhibited the NADPH-dependent peroxidation of rat microsomal and mitochondrial lipids in vitro [22]. An ethanolic extract of Radix Dipsaci enhanced the antioxidant status of blood and liver in rodents [23] and a Radix Morindae extract increased blood antioxidant enzyme activities in diabetic rats [24]. Phenylethanoids isolated from Herba Cistanche were found to prevent cell damage induced by in vitro and in vivo exposure to carbon tetrachloride in rats [25]. A recent study from our laboratory indicated that pretreatment with the methanolic extract of Herba Cistanche protected against ischemiareperfusion injury in rat hearts ex vivo and enhanced mitochondrial ATP generation in the rat hearts ex vivo and H9c2 cells in situ. The ATP-stimulating action was possibly due to enhanced oxidative phosphorylation caused by increases in the activities of complexes I and III [26]. As good body function requires a large amount of energy and antioxidant defense is essential in sustaining mitochondrial ATP production [27], the antioxidant activities of Yang herbs may safeguard ATP generation, particularly under conditions of upregulated cellular activities.

\section{Antioxidant activities of Yin tonifying herbs}

Methanolic extracts of both Fructus Ligustri and Herba Ecliptae were found to enhance hepatic glutathione (GSH) regeneration capacity in rats $[4,28]$. The enhancement of 
Table 2: Antioxidant and immunomodulatory activities of Chinese tonifying herbs

\begin{tabular}{|c|c|c|c|}
\hline & $\begin{array}{l}\text { aDPPH radical scavenging IC50 } \\
(\mathrm{mg} / \mathrm{ml})\end{array}$ & blmmunomodulatory index in vitro & Immunomodulatory index ex vivo \\
\hline Control & & $1.00 \pm 0.03$ & $1.00 \pm 0.05$ \\
\hline \multicolumn{4}{|l|}{ Yang herbs } \\
\hline Cortex Eucommiae & $>5$ & $0.46 \pm 0.02 *$ & $1.04 \pm 0.09$ \\
\hline Fructus Psoraleae & $1.0 \pm 0.0$ & $1.42 \pm 0.02 *$ & $1.23 \pm 0.01 *$ \\
\hline Herba Cistanches & $1.8 \pm 0.0$ & $0.75 \pm 0.13$ & $0.95 \pm 0.12$ \\
\hline Herba Epimedii & $1.1 \pm 0.1$ & $0.57 \pm 0.03 *$ & $0.98 \pm 0.01$ \\
\hline Radix Dipsaci & $0.8 \pm 0.0$ & $0.42 \pm 0.08^{*}$ & $1.02 \pm 0.02$ \\
\hline Radix Morindae & $>5$ & $2.16 \pm 0.10^{*}$ & $\mid .3 \mathrm{I} \pm 0.04 *$ \\
\hline Rhizoma Cibotii & $0.6 \pm 0.0$ & $0.16 \pm 0.03 *$ & $0.97 \pm 0.04$ \\
\hline Rhizoma Drynariae & $>5$ & $0.59 \pm 0.09 *$ & $0.98 \pm 0.08$ \\
\hline \multicolumn{4}{|l|}{ Yin herbs } \\
\hline Fructus Ligustri & $0.5 \pm 0.0$ & $1.73 \pm 0.07^{*}$ & $1.80 \pm 0.17^{*}$ \\
\hline Herba Dendrobii & $1.4 \pm 0.1$ & $2.54 \pm 0.09 *$ & $1.59 \pm 0.09 *$ \\
\hline Herba Ecliptae & $>5$ & $1.65 \pm 0.02 *$ & $1.27 \pm 0.12^{*}$ \\
\hline Radix Asparagi & $>5$ & $0.70 \pm 0.02 *$ & $1.24 \pm 0.05^{*}$ \\
\hline Radix Ophiopogonis & $>5$ & $1.65 \pm 0.05^{*}$ & $1.44 \pm 0.11 *$ \\
\hline Radix Oryzae & $>5$ & $0.78 \pm 0.13$ & $0.97 \pm 0.04$ \\
\hline Rhizoma Polygonati & $3.5 \pm 0.3$ & $1.43 \pm 0.09 *$ & $1.21 \pm 0.06 *$ \\
\hline Semen Prinsepiae & $>5$ & $1.70 \pm 0.03 *$ & $1.39 \pm 0.11 *$ \\
\hline
\end{tabular}

aMethanol extracts of tonifying herbs were subjected to the DPPH assay. Values given are means \pm S.D., $(n=3)$. (DPPH scavenging activity was regarded as negligible if the $\mathrm{IC}_{50}$ was $>5 \mathrm{mg} / \mathrm{ml}$ ).

bSplenocytes isolated from mice were cultured in 96-well microtiter plates in a final volume of $100 \mu \mathrm{l}$ of culture medium, with the respective methanol extracts added at final concentrations ranging from $15.6-1000 \mu \mathrm{g} / \mathrm{ml}$. Values given are means \pm S.E.M., $(n=4)$.

cAnimals were pretreated orally with the methanol extracts at a daily dose of I g/kg for 3 days. All animals were sacrificed 24 hours post-dosing. Splenocytes isolated from pretreated animals were cultured in microtiter plates in a final volume of $100 \mu \mathrm{l}$ culture medium. Values given are means \pm S.E.M., $(n=3-5)$.

$*$ Significantly different from the control group $(P<0.05)$

hepatic GSH regeneration capacity by Fructus Ligustri was associated with a hepatoprotective action against carbon tetrachloride toxicity [28]. Activity-directed fractionation of Fructus Ligustri indicated that the hepatoprotective principle(s) resided mainly in the oleanolic acid-enriched butanol and chloroform fractions [28]. Moreover, our recent studies showed that both short and long term pretreatment with oleanolic acid protected against myocardial ischemia-reperfusion injury in rats $[29,30]$. It was suggested that the cardioprotection afforded by oleanolic acid pretreatment was related to the enhancement of mitochondrial antioxidant mechanism mediated by GSH and $\alpha$-tocopherol [29]. Both experimental and clinical investigations indicated that the antioxidant status influenced immunocompetence, particularly under conditions of stress such as physical exercises or chronic diseases [31]. The antioxidant activities of Yin tonifying herbs may positively influence immunostimulatory activities.

\section{Experimental studies on a 'Yang-invigorating' herbal formula}

A 'Yang-invigorating' herbal formula named VI-28 has been shown to produce 'Yang-invigorating' effects [32] and enhance red cell antioxidant status, particularly $\mathrm{Cu}$ Zn-superoxide dismutase (SOD) activity, in elderly male human subjects [33]. This herbal formula is comprised of
Radix Ginseng, Cornu Cervi, Cordyceps, Semen Allii, Fructus Cnidii, Fructus Evodiae and Rhizoma Laemferiae. Recently we investigated the effects of long-term VI-28 treatment on red cell Cu-Zn-SOD activity, mitochondrial functional ability, and antioxidant levels, in various tissues of rats of both sexes [34]. The results indicated that VI-28 treatment increased red cell $\mathrm{Cu}-\mathrm{Zn}$-SOD activity and mitochondrial ATP generation capacity, increased the levels of reduced GSH and $\alpha$-tocopherol, and reduced Mn-SOD activities. The enhancement of ATP generation by VI-28 increased mitochondrial ROS production, resulting in the upregulation of mitochondrial antioxidant mechanism. The VI-28induced increase in mitochondrial antioxidant capacity in various tissues was evidenced by a significant reduction in ROS generation. Given that cellular energy status and mitochondrial ROS generation are factors critically involved in aging, the dual effect of 'Yang-invigoration' produced by VI-28 may have clinical implications in the prevention of age-related diseases.

\section{Immunomodulatory activities of Yin tonifying herbs}

It was suggested that the proper functioning of the immune system requires dynamic interactions between Yang and Yin. And while the antigen-nonspecific immune response is associated with Yang, the antigen-specific response is related toYin [35]. One of our earlier studies 
Table 3: Nomenclature and classification of selected Yang and Yin tonifying herbs *

\begin{tabular}{|c|c|c|}
\hline Pharmaceutical name (Chinese pinyin) & Plant part used & Latin botanical name \\
\hline \multicolumn{3}{|l|}{ Yang tonifying herbs } \\
\hline Cortex Eucommiae (Duzhong) & Bark & Eucommia ulmoides Oliv. \\
\hline Fructus Psoraleae (Buguzhi) & Fruit & Psoralea corylifolia L. \\
\hline Herba Cistanches (Roucongrong) & Whole plant & Cistanche salsa (C.A. Meyer) G. Beck. \\
\hline Herba Epimedii (Yinyanghuo) & Whole plant & Epimedium grandiflorum Morr. \\
\hline Radix Dipsaci (Xuduan) & Root & Dipsacus japonicus Miq. \\
\hline Radix Morindae (Bajitian) & Root & Morinda officinalis How \\
\hline Rhizoma Cibotii (Gouji) & Rhizome & Cibotium barometz (L.) J. Sm. \\
\hline Rhizoma Drynariae (Gusuibu) & Rhizome & Drynaria fortunei (Kunze) J. Sm. \\
\hline Semen Cuscutae (Tusizi) & Seed & Cuscuta chinensis Lam. \\
\hline Herba Cynomorii (Suoyang) & Whole plant & Cynomorium songricum Rupr. \\
\hline \multicolumn{3}{|l|}{ Yin tonifying herbs } \\
\hline Fructus Ligustri (Nuzhenzi) & Fruit & Ligustrum lucidum Ait. \\
\hline Herba Dendrobii (Shihu) & Whole plant & Dendrobium nobile Lindl. \\
\hline Herba Ecliptae (Mohanlian) & Whole plant & Ecliptae prostrata L. \\
\hline Radix Asparagi (Tianmendong) & Root & Asparagus cochinchinensis (Lour.) Merr. \\
\hline Radix Ophiopogonis (Maimendong) & Root & Ophiopogon japonicus (L. f.) Ker-Gawl. \\
\hline Radix Oryzae (Nuodaogenxu) & Root & Oryza sativa $\mathrm{L}$. \\
\hline Rhizoma Polygonati (Yuzhu) & Rhizome & Polygonatum odoratum (Mill.) Druce \\
\hline Semen Prinsepiae (Ruiren) & Seed & Prinsepia uniflora Batal. \\
\hline Semen Sesami (Heizhima) & Seed & Sesamum indicum L. \\
\hline \multicolumn{3}{|l|}{ 'Fu Zheng' herb } \\
\hline Ganoderma (Lingzhi) & Fruiting body & Ganoderma lucidum (Leyss. Ex Fr.) Karst \\
\hline \multicolumn{3}{|l|}{ Yin-Yang tonifying herb } \\
\hline Cordyceps (Dongchongxiacao) & Whole plant & Cordycep sinensis \\
\hline
\end{tabular}

*Adapted from Liang [17]

investigated antioxidant and immunomodulatory activities in different categories of tonifying herbs. The results showed that 6 and 7 of a total of 8 Yin herbs tested potentiated concanavalin A (Con A)-stimulated splenocyte proliferation (an antigen-specific response) in mice in vitro and ex vivo respectively. By contrast, only 3 of 9 Yang herbs tested showed a similar enhancement of the Con A-stimulated immune response [4] (Table 2).

Among the Yin herbs, the methanolic extract of Fructus Ligustri yielded the most robust immunostimulatory action in mouse splenocytes [4]. Differential extraction of Fructus Ligustri by solvents of increasing polarity indicated that the immunostimulatory activity resided mainly in the petroleum ether fraction [36]. Oleanolic acid, an immunomodulatory triterpenoid commonly found in herbs including Fructus ligustri $[37,38]$, was undetectable in this fraction [36]. Currently, activity-directed fractionation of the petroleum ether extract of Fructus Ligustri is under way in our laboratory. Various immunomodulatory actions of Yin tonifying herbs, and the active ingredients of the herbs, have been reported in other studies (Table 5). An

Table 4: Antioxidant activities of Yang tonifying herbs

\begin{tabular}{|c|c|c|}
\hline HERBS & ANTIOXIDANT ACTIVITIES & REFS \\
\hline Herba Epimedii & water extract caused development of superoxide scavenging activity & {$[18]$} \\
\hline Rhizoma Drynariae & $\begin{array}{l}\text { water extract decreased oxidant production in rat osteoblasts } \\
\text { water extract caused development of inhibitory effect on biomolecular oxidative damage }\end{array}$ & {$[20]$} \\
\hline Radix Dipsaci & ethanol extract enhanced blood and liver antioxidant status in rats and mice & [23] \\
\hline Fructus Psoraleae & $\begin{array}{l}\text { active ingredients (bakuchiol, isobavachin, isobavachalcone) inhibited the NADPH-dependent peroxidation of liver } \\
\text { microsomal and mitochondrial lipids in vitro in rats }\end{array}$ & {$[22]$} \\
\hline Semen Cuscutae & acetone/water $(\mathrm{I}: \mathrm{I}, \mathrm{v} / \mathrm{v})$ extract caused development of oxygen radical scavenging activity & [16] \\
\hline Herba Cistanche & $\begin{array}{l}\text { water extract caused development of superoxide scavenging activity } \\
\text { phenylethanoids prevented cell damage induced by exposure to carbon tetrachloride in vitro and in vivo }\end{array}$ & $\begin{array}{l}{[18]} \\
{[25]}\end{array}$ \\
\hline Cortex Eucommiae & $\begin{array}{l}\text { water extract caused development of hydroxyl radical scavenging activity } \\
\text { water extract caused development of inhibitory effect on biomolecular oxidative damage }\end{array}$ & $\begin{array}{l}{[19]} \\
{[21]}\end{array}$ \\
\hline Radix Morindae & ethanol extract increased blood superoxide dismutase and catalase activities in diabetic rats & {$[24]$} \\
\hline Rhizoma Cibotii & acetone/water $(I: I, v / v)$ extract caused development of oxygen radical scavenging activity & {$[16]$} \\
\hline
\end{tabular}


aqueous extract of Radix Asparagi was found to inhibit tissue necrosis factor- $\alpha$ (TNF- $\alpha$ ) secretion by suppressing Interleukin (IL)-2 secretion from astrocytes, implicating that the extract might exhibit anti-inflammatory activity in the central nervous system [39]. Both the crude aqueous extract and the two active ingredients (ruscogenin and ophiopogonin D) of Radix Ophiopogonis produced antiinflammatory effects in rodents [40]. While the aqueous extract inhibited xylene-induced ear swelling and carrageenan-induced paw edema in mice, it also suppressed carrageenan-induced pleural leukocyte migration in rats, and the zymosan-evoked migration of peritoneal total leukocytes and neutrophils in mice. Treatments with ruscogenin and ophiopogonin D decreased zymosaninduced peritoneal leukocyte migration in mice and reduced the phorbol-12-myristate-13 acetate-induced adhesion of HL60 cells to ECV304 cells [40]. Several sesquiterpenes isolated from Herba Dendrobii were found to exhibit immunomodulatory activity by exerting comitogenic effects on Con A and lipopolysaccharide-stimulated mouse splenocytes $[41,42]$. It has recently been reported that an ethanolic extract of black rice (the fruit of Oryza sativa) showed anti-asthmatic effects in a mouse model [43]. Treatment with the ethanolic extract of black rice reduced the number of eosinophils in bronchoalveolar lavage fluid, alleviated the airway hyper-response, and decreased the extent of airway inflammation in ovalbumin (OVA)-immunized and -aerolized mice challenged with OVA. Moreover, the ethanolic extract treatment decreased interferon- $\gamma$ (INF- $\gamma$ ), IL-4, IL-5 and IL-13 levels in the supernatants of cultured splenocytes and suppressed the plasma levels of OVA-specific immunoglobulin (Ig)G, IgG2 $\alpha$, IgG1 and total IgE in OVA-immunized and -challenged mice [43]. Clinical investigations indicated that intramuscular injection of undiluted Fructus Ligustri extract at a dose of 2-4 $\mathrm{ml}$ once or twice daily could prevent leucopenia caused by chemotherapy or radiotherapy. Fructus Ligustri treatment normalized white blood cell counts, thereby increasing tolerance to chemo/ radiotherapy [44]. Oral administration of Fructus Ligustri tablets at a daily dose of $50 \mathrm{~g}$ equivalence of crude herb was found to ameliorate the symptoms of chronic bronchitis [44]. A herbal formula comprising Fructus Ligustri, Radix Scutellariae, Radix Astragalus and Eupolyphaga et polyphae was found to alleviate symptoms and improve immune function in HIV/AIDS patients [45].

\section{Ganoderma - A 'Fu Zheng' tonifying herb}

Ganoderma, another Yin tonifying herb with immunomodulatory effects, is widely consumed by the Chinese people who believe that it promotes health and longevity, lowers the risk of cancer and heart diseases and boosts the immune system [46]. In Chinese medicine, Ganoderma is regarded as a very potent herb for 'Fu Zheng', a Chinese medicine concept comparable to immunotherapy/immunomodulation in Western medicine. While Ganoderma is traditionally used to increase the resistance of the body immune system to pathogens and to restore normal body functions, the herb has now also been used to decrease the side effects of Western medical procedures, such as surgery, radiotherapy and chemotherapy which often weaken the immune system. The anti-cancer/immunomodulatory effects of Ganoderma were associated with triterpenes [47], polysaccharides $[48,49]$ or immunomodulatory proteins [50] through mechanisms involving inhibition of DNA polymerase [51], inhibition of posttranslational modification of the Ras oncoprotein [52] or the stimulation of cytokine production [53]. Recent studies on the immunomodulatory activities of Ganoderma indicated that Ganoderma extract stimulated the proliferation of human peripheral blood mononuclear cells and raised the levels of mRNAs encoding Th1 and Th2 cytokines in these cells [54]. Moreover, polysaccharides of Ganoderma activated mouse splenic B cells and induced these cells to differentiate into IgM-secreting plasma cells. This process was dependent on the polysaccharide-mediated induction of Blimp-1, a master regulator capable of triggering a cascade of gene expression during plasmacytic differentiation [55]. In human peripheral B lymphocytes, the Ganoderma polysaccharide fraction enhanced antibody secretion and induced the production of Blimp-1

Table 5: Immunomodulatory activities of Yin tonifying herbs

\begin{tabular}{|c|c|c|}
\hline HERBS & IMMUNOMODULATORY ACTIVITIES & REFS \\
\hline Fructus Ligustri & $\begin{array}{l}\text { Methanolic extract or petroleum ether fraction enhanced Con A-stimulated proliferation of mouse splenocytes in } \\
\text { vitro and ex vivo }\end{array}$ & {$[4,36]$} \\
\hline Radix Asparagi & Water extract inhibited TNF- $\alpha$ secretion by suppressing IL-2 secretion from astrocytes & [39] \\
\hline Radix Ophiopogonis & $\begin{array}{l}\text { Water extract inhibited xylene-induced ear swelling and carrageenan-induced paw edema in mice Active ingredients } \\
\text { (ruscogenin and ophiopogonin D) decreased zymosan-induced adhesion of HL60 cells to ECV } 304 \text { cells }\end{array}$ & {$[40]$} \\
\hline Herba Dendrobii & $\begin{array}{l}\text { Active ingredients (sesquiterpenes) showed a co-mitogenic effect on Con A and lipopolysaccharide-stimulated } \\
\text { mouse splenocytes }\end{array}$ & {$[41,42]$} \\
\hline Radix Oryza & $\begin{array}{l}\text { Ethanolic extract of black rice (the fruit of Oryza sativa) decreased the extents of airway inflammation and hyper- } \\
\text { response in OVA-immunized and aerolized OVA-challenged mice } \\
\text { Ethanolic extract of black rice decreased various cytokine levels in the supernatant of cultured splenocytes and } \\
\text { suppressed the plasma levels of OVA-specific lgG and total IgE in OVA-immunized and challenged mice }\end{array}$ & {$[43]$} \\
\hline
\end{tabular}




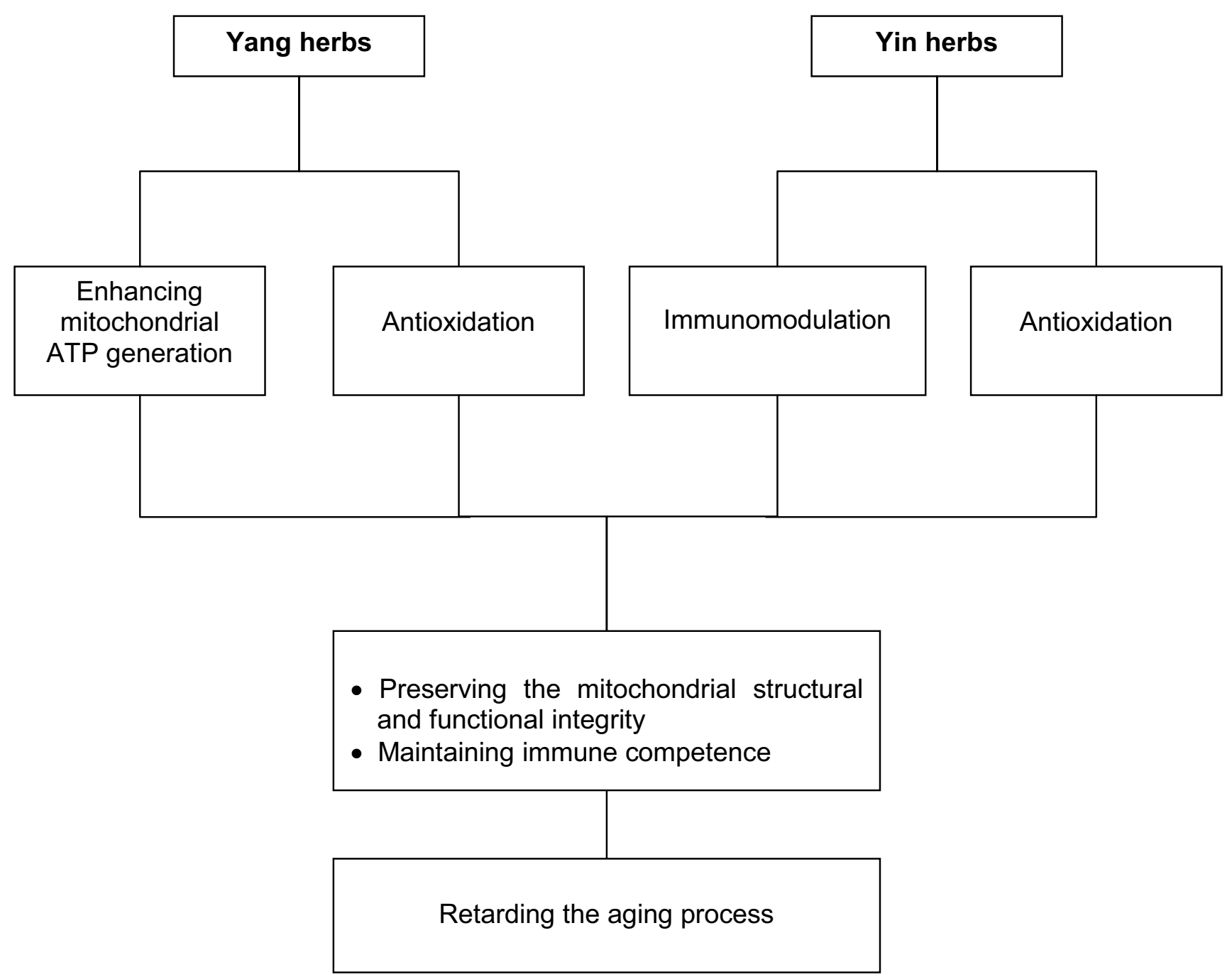

Figure I

Anti-aging potential of Chinese tonifying herbs.

mRNA, though it failed to induce lymphocyte differentiation [55].

In addition to immunomodulating activities, Ganoderma possesses in vivo antioxidant potential, another aspect of Yin tonifying action. Treatment with Ganoderma extract was found to enhance the hydroxyl radical scavenging activity of rabbit blood plasma [56,57]. Ganoderma acted by stimulating cellular and mitochondrial SOD activities, thereby enhancing the antioxidant capacity of the body [58]. It was shown that an intraperitoneal injection of Ganoderma extract following a lethal dose of cobalt X-ray radiation caused a marked prolongation of survival time in mice [59]. Pretreatment with Ganoderma extract also markedly protected against carbon tetrachloride-induced hepatic damage and the associated impairment in hepatic antioxidant status [60].

\section{Cordyceps - A Yin/Yang tonifying herb}

Cordyceps, a premium Chinese tonifying herb which replenishes the 'kidney' and soothes the 'lung', is prescribed for the treatment of a host of disorders, including hyposexualities, hyperglycemia, hyperlipidemia, asthenia after illness, respiratory diseases, renal disorders, liver and heart diseases [61]. Cordyceps is regarded as a tonifying herb with both 'Yin-nourishing' and 'Yang-invigorating' actions. Pharmacological studies have shown that Cordyceps possesses a wide spectrum of biological activities including antioxidation [61-64], immunopotentiation [65-68], anti-tumorigenesis [68-71], anti-inflammation 
[72] and stimulation of testosterone biosynthesis [73]. We have recently investigated the effects of wild and cultured Cordyceps on Con A-stimulated splenocytes (an in vitro bioassay for Yin tonifying action) and myocardial ATP generation capacity (an ex vivo bioassay for Yang tonifying action) [74]. The results indicated that methanolic extracts of wild and cultured Cordyceps enhanced both the Con A-stimulated splenocyte proliferation in vitro and myocardial mitochondrial ATP generation ex vivo in mice, with no significant difference in potencies when the two types of Cordyceps were compared. While the immunopotentiating effect was associated with an increase in IL2 production, the stimulation of myocardial ATP generation was paralleled by an enhancement in mitochondrial electron transport. When compared with typical Yin and Yang tonifying herbs (Fructus Ligustri and Herba Cynomorii respectively), Cordyceps was found to possess both Yin and Yang tonifying actions, with a lower potency in both modes of action. The observation of both immunopotentiating and ATP-enhancing activities in Cordyceps extracts further supports the pharmacological basis of Yin and Yang tonifying herbs in Chinese medicine.

\section{Conclusion}

Yang tonifying herbs stimulate mitochondrial ATP generation, leading to the enhancement of cellular/mitochondrial antioxidant status, presumably through the intermediacy of ROS. Yin tonifying herbs, which also possess antioxidant properties, are mainly immunomodulatory, thereby boosting weak immune functions and suppressing overreactive or unbalanced immune responses. Cordyceps, highly regarded as a tonifying herb with a dual action of Yin and Yang, stimulates mitochondrial ATP generation and enhances cellular immune responses. Given that impairment in mitochondrial functional ability and antioxidant status, and a decline in immunocompetence, are believed to be critically involved in the development of age-related diseases and the aging process, the abilities of Yang and Yin tonifying herbs to enhance ATP generation capacity and to produce antioxidant and immunomodulatory actions are beneficial for safeguarding health and delaying the onset of senility (Figure 1). While animal models may be used for testing working hypotheses on Yang and Yin tonifying actions, clinical studies, using Yang and Yin tonifying herbs and/ or defined chemicals isolated from the herbs or synthesized in the laboratory, on age-related variations in antioxidant and immune function, would be of considerable value.

\section{List of abbreviations}

ATP: adenosine triphosphate

Con A: concanavalin A
GSH: reduced glutathione

Ig: immunoglobulin

IL: interleukin

INF: interferon

OVA: ovalbumin

ROS: reactive oxygen species

SOD: superoxide dismutase

TNF: tissue necrosis factor

\section{Competing interests}

The author(s) declare that they have no competing interests.

\section{Authors' contributions}

KMK conceived and wrote the article. HYL did literature research and organized the information.

\section{References}

I. Moskovitz J, Yim MB, Chock PB: Free radicals and disease. Arch Biochem Biophys 2002, 397:354-359.

2. Hakim FT, Flomerfelt FA, Boyiadzis M, Gress RE: Aging, immunity and cancer. Curr Opin Immunol 2004, 16:151-156.

3. Boren E, Gershwin ME: Inflamm-aging: autoimmunity, and the immune-risk phenotype. Autoimmun Rev 2004, 3:40I-406.

4. Yim TK, Ko KM: Antioxidant and immunomodulatory activities of Chinese tonifying herbs, in vitro and ex vivo. Pharmaceut Biol 2002, 40:329-335.

5. Szeto YT, Benzie IFF: Is the yin-yang nature of Chinese herbal medicine equivalent to antioxidation - oxidation? J Ethnopharmacol 2006, 108:36|-366

6. Jin R, Wan LL, Mitsuishi T, Sato S, Akuzawa Y, Kodama K, Kurashige $S$ : Effect of ski-ka-ron and Chinese herbs on cytokine production of macrophage in immunocompromised mice. Am J Chin Med 1994, 22:255-266.

7. State Administration of Traditional Chinese Medicine and Pharmacy Tonics. In Advanced Textbook on Traditional Chinese Medicine and Pharmacology Volume 7. Beijing: New World Press; 1995:193-213.

8. State Administration of Traditional Chinese Medicine and Pharmacy: Yin and Yang and the Five Elements. In Advanced Textbook on Traditional Chinese Medicine and Pharmacology Volume 6. Beijing: New World Press; 1995: 165-180.

9. Taitano K, Schnyer R, Allen JJB, Manber R, Hitt SK: The psychophysiology of yin and yang. J Herbal Pharmacother 2003, 3:63.

10. Kong YC: The Cultural Fabrics of Chinese Medicine. Hong Kong: Commercial Press; 2005: I-I I.

II. Ko KM, Leon TY, Mak DHF, Chiu PY, Du Y, Poon MKT: A characteristic pharmacological action of 'Yang-invigorating' Chinese tonifying herbs: Enhancement of myocardial ATP. generation capacity. Phytomedicine 2006, 13:636-642.

12. Leeuwenburgh C, Hansen PA, Holloszy JO, Heinecke JW: Hydroxyl radical generation during exercise increases mitochondrial protein oxidation and levels of urinary dityrosine. Free Radic Biol Med 1999, 27:186-192.

13. Chandwaney R, Leichtweis S, Leeuwenburgh C, Ji LL: Oxidative stress and mitochondrial function in skeletal muscle: Effects of aging and exercise training. Age 1998, 21:109-117.

14. Halliwell B: Antioxidants in human health and disease. Annu Rev Nutr 1996, 16:33-50.

15. Finkel T, Holbrook NJ: Oxidants, oxidative stress and the biology of ageing. Nature 2000, 408:239-247. 
16. Ou B, Huang D, Hampsch-Woodill M, Flanagan JA: When east meets west: the relationship between yin-yang and antioxidation-oxidation. FASEB J 2003, I 7:127-129.

17. Liang SM: Formularies of Chinese Materia Medica (Ist Edition). Guangdong Science and Technology Press; 1991:637-663.

18. Liu W, Ogata T, Sato S, Unoura K, Onodera J: Superoxide scavenging activities of sixty Chinese medicines determined by an ESR spin-trapping method using electrogenerated superoxide. Yakugaku Zasshi 200I, I 2 I:265-270.

19. Yen GC, Hsieh CL: Reactive oxygen species scavenging activity of Du-zhong (Eucommia ulmoides oliv.) and its active compounds. I Agric Food Chem 2000, 48:3431-3436.

20. Liu HC, Chen RM, Jian WC, Lin YL: Cytotoxic and antioxidant effects of the water extract of traditional Chinese herb gusuibu (Drynaria fortunei) on rat osteoblasts. J Formos Med Assoc 2001, 100:383-388.

21. Hsieh CL, Yen GC: Antioxidant actions of du-zhong (Eucommia ulmoides Oliv.) toward oxidative damage in biomolecules. Life Sci 2000, 66: I387-I400.

22. Haraguchi H, Inoue J, Tamura $\mathrm{Y}$, Mizutani $\mathrm{K}$ : Antioxidative components of Psoralea corylifolia (Leguminosae). Phytother Res 2002, I 6:539-544.

23. Wong YT, Wong KK, Yeung K, Mo G: Pharmacology and clinical research on Dipsacus asperoides. Pharmacol Clin Chin Materia Medica 1996, 3:20-24.

24. Soon YYTB: Evaluation of the hypoglycemic and anti-oxidant activities of Morinda officinalis in streptozotocin-induced diabetic rats. Singapore Med J 2002, 43:77-85.

25. Xiong Q, Hase K, Tezuka Y, Tani T, Namba T, Kadota S: Hepatoprotective activity of phenylethanoids from Cistanche deserticola. Planta Med 1998, 64: I20-125.

26. Leung HY: Effect of Herba Cistanche on mitochondrial ATP generation: A pharmacological basis of 'Yang-invigoration'. In MPhil thesis Hong Kong University of Science \& Technology, Department of Biochemistry; 2006.

27. Melov S: Therapeutics against mitochondrial oxidative stress in animal models of aging. Ann N Y Acad Sci 2002, 959:330-340.

28. Yim TK, Wu WK, Pak WF, Ko KM: Hepatoprotective action of an oleanolic acid-enriched extract of Ligustrum lucidum fruits is mediated through an enhancement on hepatic glutathione regeneration capacity in mice. Phytother Res 2001 I 5:589-592.

29. Du Y, Ko KM: Effects of pharmacological preconditioning by emodin/oleanolic acid treatment and/or ischemic preconditioning on mitochondrial antioxidant components as well as the susceptibility to ischemia-reperfusion injury in rat hearts. Mol Cell Biochem 2006, 288: I35-142.

30. Du Y, Ko KM: Oleanolic acid protects against myocardia ischemia-reperfusion injury by enhancing mitochondrial antioxidant mechanism mediated by glutathione and alphatocopherol in rats. Planta Med 2006, 72:222-227.

31. Hemila $\mathrm{H}$ : The effect of nutrition on exercised-induced immunodepression. Nutr Rev 2006, 64:119-131.

32. Chui SH, Ko KM: Biochemical, immunological, hormonal and anti-oxidant effects of Chinese herbal suppository, Vigconic HY28, on male subjects - an anti-aging solution? [abstract]. Proceedings of Anti-aging World Conference: 2003; Paris 2003: 105.

33. Mak DHF, Chiu PY, Poon MKT, Ng TT, Chung YK, Lam BYH, Du Y, Ko KM: A yang-promoting Chinese herbal suppository preparation enhances the antioxidant status of red cells in male human subjects. Phytother Res 2004, 18:525-530.

34. Leung HY, Chiu PY, Poon MK, Ko KM: A yang-invigorating Chinese herbal formula enhances mitochondrial functional ability and antioxidant capacity in various tissues of male and female rats. Rejuven Res 2005, 8:238-247.

35. Chen H: Scientific Basis of Chinese Medicine Hong Kong: Commercial Press; 1991.

36. Yim TK, Ko KM: Effects of Fructus Ligustri Lucidi extracts on concanavalin A-stimulated proliferation of isolated murine splenocytes in vitro and ex vivo. Pharmaceut Biol 200I, 39:|46-|5|.

37. Liu J: Oleanolic acid and ursolic acid: research perspectives. Ethnopharmacol 2005, 100:92-94.

38. Ha CL, Weng CY, Wang L, Lian TW, Wu MJ: Immunomodulatory effect of Glossogyne tenuifolia in murine peritoneal macrophages and splenocytes. J Ethnopharmacol 2006, I07: I I6-125.
39. Kim H, Lee E, Lim T, Jung J, Lyu Y: Inhibitory effect of Asparagus cochinchinensis on tumor necrosis factor-alpha secretion from astrocytes. Int J Immunopharmacol I998, 20:153-162.

40. Kou J, Sun Y, Lin Y, Cheng Z, Zheng W, Yu B, Xu Q: Anti-inflammatory activities of aqueous extract from Radix Ophiopogon japonicus and its two constituents. Biol Pharm Bull 2005, 28: | $234-1238$.

4I. Zhao W, Ye Q, Tan X, Jiang H, Li X, Chen K, Kinghom AD: Three new sesquiterpene glycosides from Dendrobium nobile with immunomodulatory activity. J Nat Prod 200 I, 64: I I 96-I 200.

42. Ye Q, Qin G, Zhao W: Immunomodulatory sesquiterpene glycosides from Dendrobium nobile. Phytochemistry 2002, 6 I:885-890.

43. Lee SH, Sohn YS, Kang KK, Kwon JW, Yoo M: Inhibitory Effect of DA-920 I, an Extract of Oryza sativa L., on Airway Inflammation and Bronchial Hyperresponsiveness in Mouse Asthma Model. Biol Pharm Bull 2006, 29: I I48-I I 53.

44. Chang HM, But PPH, (Eds): Nuzhensi. In Pharmacology and Applications of Chinese Materia Medica Volume I. Singapore: World Scientific Publishing Co Pte Ltd; 1986: I34.

45. Wei JA, Sun LM, Chen YX: Effects of ailing granule on immunoreconstruction in HIVIAIDS patients. Zhongguo Zhongxiyi Jiehe Zazhi 2006, 26:319-321.

46. Chang ST, Buswell JA: Ganoderma lucidum - a mushrooming medicinal mushroom. Int J Medicinal Mushrooms I999, I: I39- I46.

47. Min BS, Gao J], Nakamura N, Hattori M: Triterpenes from the spores of Ganoderma lucidum and their cytotoxicity against meth-A and LLC tumor cells. Chem Pharm Bull (Tokyo) 2000 48: $1026-1033$.

48. Miyazaki T, Nishijima M: Studies on fungal polysaccharides. XXVII. Structural examination of a water-soluble, antitumor polysaccharide of Ganoderma lucidum. Chem Pharm Bull (Tokyo) 1981, 29:36II-3616.

49. Sone Y, Okuda R, Wada N, Kisida E Misuki A. Structures and antitumor activities of the polyoacchardes isolated from fruiting body and the growing culture of mcelium of Ganoderma lucidum. Agric Biol Chem 1995, 49:2641-2653.

50. Lin WH, Hung $\mathrm{CH}$, Hsu Cl, Lin JY: Dimerization of the N-terminal amphipathic alpha-helix domain of the fungal immunomodulatory protein from Ganoderma tsugae (Fip-gts) defined by a yeast two-hybrid system and site-directed mutagenesis. J Biol Chem 1997, 272:20044-20048.

5I. Mizushina Y, Hanashima L, Yamaguchi T, Takemura M, Sugawara F, Saneyoshi M, Matsukage A, Yoshida S, Sakaguchi K: A mushroom fruiting body-inducing substance inhibits activities of replicative DNA polymerases. Biochem Biophys Res Commun 1998 , 249: 17-22.

52. Lee S, Park S, Oh JW, Yang C: Natural inhibitors for protein prenyl-transferases. Planta Med 1998, 64:303-308.

53. Wang SY, Hsu ML, Hsu HC, Tzeng CH, Lee SS, Shiao MS, Ho CK: The anti-tumor effect of Ganoderma lucidum is mediated by cytokines released from activated macrophages and $T$ lymphocytes. Int / Cancer 1997, 70:699-705.

54. Chan WK, Lam DT, Law HK, Wong WT, Koo MW, Lau AS, Lau YL, Chan GC: Ganoderma lucidum mycelium and spore extracts as natural adjuvants for immunotherapy. I Altern Complement Med 2005, I I:I047-1057.

55. Lin KI, Kao YY, Kuo HK, Yang WB, Chou A, Lin HH, Au AL, Wong $\mathrm{CH}$ : Reishi polysaccharides induce immunoglobulin production through the TLR4/TLR2-mediated induction of transcription factor blimp-I. J Biol Chem 28 I:24 I I I-24I 23.

56. Wang J, Zhang J, Chen W: Study of the action of Ganoderma lucidum on scavenging hydroxyl radical from plasma. J Trad Chin Med 1 985, 5:55-60.

57. Wachtel-Galor S, Tomlinson B, Benzie IF: Ganoderma lucidum ("Lingzhi"), a Chinese medicinal mushroom: biomarker responses in a controlled human supplementation study. $\mathrm{Br}$ J Nutr 2004, 91 :263-269.

58. Dai YR, Gao CM, Tian QL, Yin Y: Effect of extracts of some medicinal plants on superoxide dismutase activity in mice. Planta Med 1987, 53:309-310.

59. Chang HM, But PPH, (Eds): Lingzhi. In Pharmacology and Applications of Chinese Materia Medica I Singapore: World Scientific Publishing Co Pte Ltd; 1986:642-653. 
60. $\mathrm{Ng}$ PC, So CM, Yick PK, Kong YC, Ko KM: Antioxidant activity of Ganoderma lucidum: Protective effect on carbon tetrachloride-induced hepatotoxicity. Acta Horticulture 1993, 32:219-225.

6I. Zhu JS, Halpern GM, Jones K: The scientific rediscovery of an ancient Chinese herbal medicine: Cordyceps sinensis: part I. J Altern Complement Med 1998, 4:289-303.

62. Yamaguchi $Y$, Kagota S, Nakamura K, Shinozuka K, Kunitomo M: Antioxidant activity of the extracts from fruiting bodies of cultured Cordyceps sinensis. Phytother Res 2000, 14:647-649.

63. Li SP, Li P, Dong TT, Tsim KW: Anti-oxidation activity of different types of natural Cordyceps sinensis and cultured Cordyceps mycelia. Phytomedicine 200I, 8:207-2I 2 .

64. Li SP, Li P, Dong TT, Tsim KW: Determination of nucleosides in natural Cordyceps sinensis and cultured Cordyceps mycelia by capillary electrophoresis. Electrophoresis 200I, 22: I44-I50.

65. Gong M, Xhu Q, Wang XL, Ma JX, Zhang WJ: Molecular structure and immunoactivity of the polysaccharide from Cordyceps sinensis (Berk.) Sacc. China Biochem J 1990, 6: I 44-I50.

66. Xu RH, Peng XE, Chen GZ, Chen GL: Effects of Cordyceps sinensis on natural killer activity and colony formation of $\mathrm{B} / 6$ melanoma. Chin Med J (Engl) 1992, I05:97-101.

67. Chen GZ, Chen GL, Su T, Hsieh GC, Henshall JM: Effects of Cordyceps sinensis on murine $\mathbf{T}$ lymphocyte subsets. Chin Med J (Engl) 199I, 104:4-8.

68. Shin KH, Lim SS, Lee S, Lee YS, Jung SH, Cho SY: Anti-tumour and immuno-stimulating activities of the fruiting bodies of Paecilomyces japonica, a new type of Cordyceps spp. Phytother Res 2003, I 7:830-833.

69. Ohmori T, Tamura K, Tsuru S, Nomoto K: Antitumor activity of protein-bound polysaccharide from Cordyceps ophioglossoides in mice. Jpn I Cancer Res 1986, 77: |256-1263.

70. Yoshida J, Takamura S, Yamaguchi N, Ren LJ, Chen H, Koshimura S, Suzuki S: Antitumor activity of an extract of Cordyceps sinensis (Berk.) Sacc. against murine tumor cell lines. Jpn J Exp Med 1989, 59:|157-161.

7I. Chen YJ, Shiao MS, Lee SS, Wang SY: Effect of Cordyceps sinensis on the proliferation and differentiation of human leukemic U937 cells. Life Sci 1997, 60:2349-2359.

72. Kim KM, Kwon YG, Chung HT, Yun YG, Pae HO, Han JA, Ha KS, Kim TW, Kim YM: Methanol extract of Cordyceps pruinosa inhibits in vitro and in vivo inflammatory mediators by suppressing NF-kappaB activation. Toxicol Appl Pharmacol 2003, 190: I-8.

73. Huang BM, Hsu CC, Tsai SJ, Sheu CC, Leu SF: Effects of Cordyceps sinensis on testosterone production in normal mouse Leydig cells. Life Sci 2001, 69:2593-2602.

74. Siu KM, Mak DH, Chiu PY, Poon MK, Du Y, Ko KM: Pharmacological basis of 'Yin-nourishing' and 'Yang-invigorating' actions of Cordyceps, a Chinese tonifying herb. Life Sci 2004, 76:385-395.

Publish with Bio Med Central and every scientist can read your work free of charge

"BioMed Central will be the most significant development for disseminating the results of biomedical research in our lifetime. "

Sir Paul Nurse, Cancer Research UK

Your research papers will be:

- available free of charge to the entire biomedical community

- peer reviewed and published immediately upon acceptance

- cited in PubMed and archived on PubMed Central

- yours - you keep the copyright

Submit your manuscript here:

http://www.biomedcentral.com/info/publishing_adv.asp
BioMedcentral 